\title{
Results of endoscopic surgery and intralesional steroid therapy for airway compromise due to tracheobronchial Wegener's granulomatosis
}

\author{
S A R Nouraei, ${ }^{1}$ R Obholzer, ${ }^{1}$ P W Ind, ${ }^{2}$ A D Salama ${ }^{3}$ C D Pusey, ${ }^{3}$ F Porter, ${ }^{4}$ \\ D J Howard, ${ }^{1}$ G S Sandhu'
}

- Full details of the surgical and anaesthetic techniques are published online only at http:// thorax.bmi.com/content/vol63/ issue1

${ }^{1}$ Department of Ear Nose and Throat Surgery, Charing Cross Hospital, London, UK; ${ }^{2}$ Department of Respiratory Medicine, Hammersmith Hospital, London, UK;

${ }^{3}$ Department of Renal Medicine, Hammersmith Hospital, London, UK; ${ }^{4}$ Department of Anaesthesia, Charing Cross Hospital, London, UK

Correspondence to:

Dr Reza Nouraei, National Centre for Airway Reconstruction, Department of Ear Nose and Throat Surgery, Charing Cross Hospital, London W6 8RF, UK; RN@cantab.net

Presented at the European Respiratory Society (ERS) Meeting, Stockholm, Sweden (Sept 2007). An Inverventional Pulmonology Travel Award for "best presentation by an investigator who is under 35 years" was given in recognition of this work to the first author at the ERS meeting.

Received 30 January 2007 Accepted 30 May 2007 Published Online First 15 June 2007

\section{ABSTRACT}

Background: Upper airway compromise due to tracheobronchial stenosis commonly occurs in patients with Wegener's granulomatosis (WG). There is at present no consensus on the optimal management of this life threatening condition.

Objective: To assess the results of laryngo-tracheobronchoscopy, intralesional steroid therapy, laser surgery and dilatation in managing obstructive tracheobronchial WG.

Methods: Records of 18 previously untreated stridulous patients with obstructive tracheobronchial WG, treated between 2004 and 2006, were prospectively recorded on an airway database and retrospectively reviewed. Information about patient and lesion characteristics and treatment details were recorded. Treatment progress was illustrated using a timeline plot, and intervention-free intervals were calculated with actuarial analysis.

Results: There were nine males and the average age at presentation was 40 (16) years (range 13-74). There were 13 patients with tracheal and five with tracheal and bronchial lesions. The average tracheal lesion height was 8 (3) mm, located 23 (9) mm below the glottis. There were 1, 10 and 7 Myer-Cotton grade I, II and III lesions, respectively. Mean intervention-free interval following minimally invasive treatment was 26 (2.8) months. Following endobronchial therapy, the median interventionfree interval was 22 months ( $p>0.8$ vs tracheal lesions). No patient required a tracheostomy or endoluminal stenting.

Conclusions: Intralesional steroid therapy and conservative endoluminal surgery is an effective strategy for treating airway compromise due to active tracheal and bronchial WG, obviating the need for airway bypass or stenting. We recommend the combination of endotracheal dilatation, conservative laser surgery and steroid therapy as the standard of care for treating airway compromise due to obstructive tracheobronchial WG.

Wegener's granulomatosis (WG) is a multisystem autoimmune vasculitis of small to medium sized blood vessels which causes a pauci-immune necrotising granulomatous inflammation ${ }^{1}$ which can involve any organ in the body. ${ }^{2}$ The disease typically runs a relapsing and remitting course ${ }^{3}$ with many patients experiencing at least one relapse. ${ }^{4}$ The historically poor outcome of this condition ${ }^{5}$ was revolutionised following the introduction of immunosuppression with corticosteroids and cytotoxic agents. ${ }^{2}{ }^{6}$ Therapy is divided into an intensive induction phase followed by a maintenance phase once remission has been achieved. $^{26-8}$ The particular agents used are dependent on disease severity and phenotype. ${ }^{9} 10$

As many as a quarter of WG patients develop obstructive airway lesions during the course of their disease, most often in the subglottis and the proximal trachea, and less frequently in a main bronchus. ${ }^{11}$ Obstructive tracheobronchial WG, which often runs a course independent of other manifestations of the disease, can be poorly responsive to systemic therapy ${ }^{11}$ and poses a formidable therapeutic conundrum. If not promptly and effectively treated, it can lead to marked pulmonary morbidity and life threatening ventilatory and infective complications. However, over-treatment (eg, with permanent stents or by aggressive resection of lesions) can cause long term airway complications which are difficult to treat. ${ }^{11-14}$ Therefore, the challenge of managing the airway in WG lies in maintaining unimpeded ventilation and preventing infective complications during the active phase of the disease, while minimising the risk of long term iatrogenic morbidity.

In this study, we have reviewed our experience with the management of airway compromise due to active tracheobronchial WG using intralesional steroid injection, endoscopic laser photoresection and balloon dilatation.

\section{MATERIALS AND METHODS}

\section{Patient population}

Records of all patients with WG treated for acute airway compromise due to tracheobronchial obstruction between 2004 and 2006 at a tertiary and quaternary referral airway reconstruction unit were prospectively recorded on an airway database and retrospectively reviewed. Information was obtained on patient demographics, clinical presentation, lesion characteristics, number and nature of airway treatments and intervention-free-interval, defined as the length of time from one treatment episode undertaken to restore luminal patency to the next. ${ }^{15}$ To ensure uniformity of the treatment approach, eight patients who had undergone airway treatment before referral to our institution were excluded.

\section{Anaesthesia and surgery}

Procedures were performed under general anaesthesia with a total intravenous anaesthesia technique. The airway was initially secured using a supraglottic laryngeal mask, which was 
substituted in the operating room with a Dedo-Pilling suspension laryngoscope, through which high frequency supraglottic jet ventilation was delivered. The airway was visualised with a combination of microscopic and endoscopic techniques. Key steps of surgery consisted of delivering intralesional steroids, conservative laser surgery and balloon dilatation of the lesion. Full details of the surgical and anaesthetic techniques are provided in the online supplement.

\section{Data analysis}

Data were presented as means (SD) or as percentages when appropriate. Mean intervention-free interval was calculated and illustrated using the method of Kaplan and Meier, and compared with log rank statistics. Ordinal regression was used to identify predictors of the number of treatments required to achieve a sustained symptom free period. Data were analysed and displayed using SPSS release 12.0 for Windows (SPSS Inc., Chicago, Illinois, USA).

\section{RESULTS \\ Patients}

Over the study period, 18 patients with airway compromise due to active WG were treated. All patients had active granulomatous lesions obstructing the central airways with mucosal inflammation and no patient had mature fibrotic airway scars. This was assessed by endoscopic examination and confirmed by tissue biopsy where there was clinical doubt. There were equal numbers of males and females. The average age at diagnosis was 40 (16) years (range 13-74). Four patients presented with primary tracheobronchial WG while the remaining patients had an average time from diagnosis to airway treatment of 6.3 (5.3) years (range 1-23). In nine patients there were extratracheal manifestations of WG affecting the eyes, lungs, kidneys and the joints. Within the airway, the subglottis/cervical trachea were affected in all patients, and five patients had concomitant bronchial lesions. All patients had Medical Research Council Dyspnoea grades of IV or $\mathrm{V}$ at the time of surgery. The average lesion height was 7.8 (2.7) mm (range 415), and tracheal lesions were on average located 22.7 (9.5) $\mathrm{mm}$ below the glottis. There were 1, 10 and 7 Myer-Cotton grades I, II and III tracheal lesions, respectively. ${ }^{16}$ Bronchial lesions were restricted to a main bronchus in three patients and affected the ostia of one or more of the lobar bronchi in two patients. Examples of the results of treatment for both tracheal and bronchial lesions are shown in fig 1.

Eighteen patients underwent 31 procedures, with a median number of one procedure per patient (range 1-4). No patient required a tracheostomy or a tracheobronchial stent, and no open surgical procedures became necessary. No patient had significant postoperative haemorrhage or infection. Ordinal regression was undertaken to identify independent factors associated with the number of treatments each patient received. Variables entered into the model were patient age and sex, vertical height and Myer-Cotton grade of the lesion, distance from the glottis, presence of concomitant bronchial lesions and whether or not mitomycin $\mathrm{C}$ had been used at any time during treatment. No independent predictors of the number of treatment episodes per patient were identified.

The treatment timelines for all patients in the series are shown in fig 2A. The overall mean intervention-free interval following endoscopic treatment was 26.1 (SEM 2.8) months (95\% CI 19.4 to 30.7 months) (fig $2 \mathrm{~B}$ ). There was no significant difference between the durability of endotracheal and endobronchial treatments ( $>0.8$; log rank analysis). Topical mitomycin $C$ was used in seven patients with high grade or recurrent lesions. It did not significantly increase the intervention-free interval and was associated with one patient developing stridor within 1 month of treatment because of airway crusting, which was managed endoscopically with no permanent sequelae.
Figure 1 (A) Intraoperative results of treating a tracheal lesion. (B) Intraoperative results of treating a bronchial lesion. The submucosal "white streaks" are the result of intralesional steroid injection.
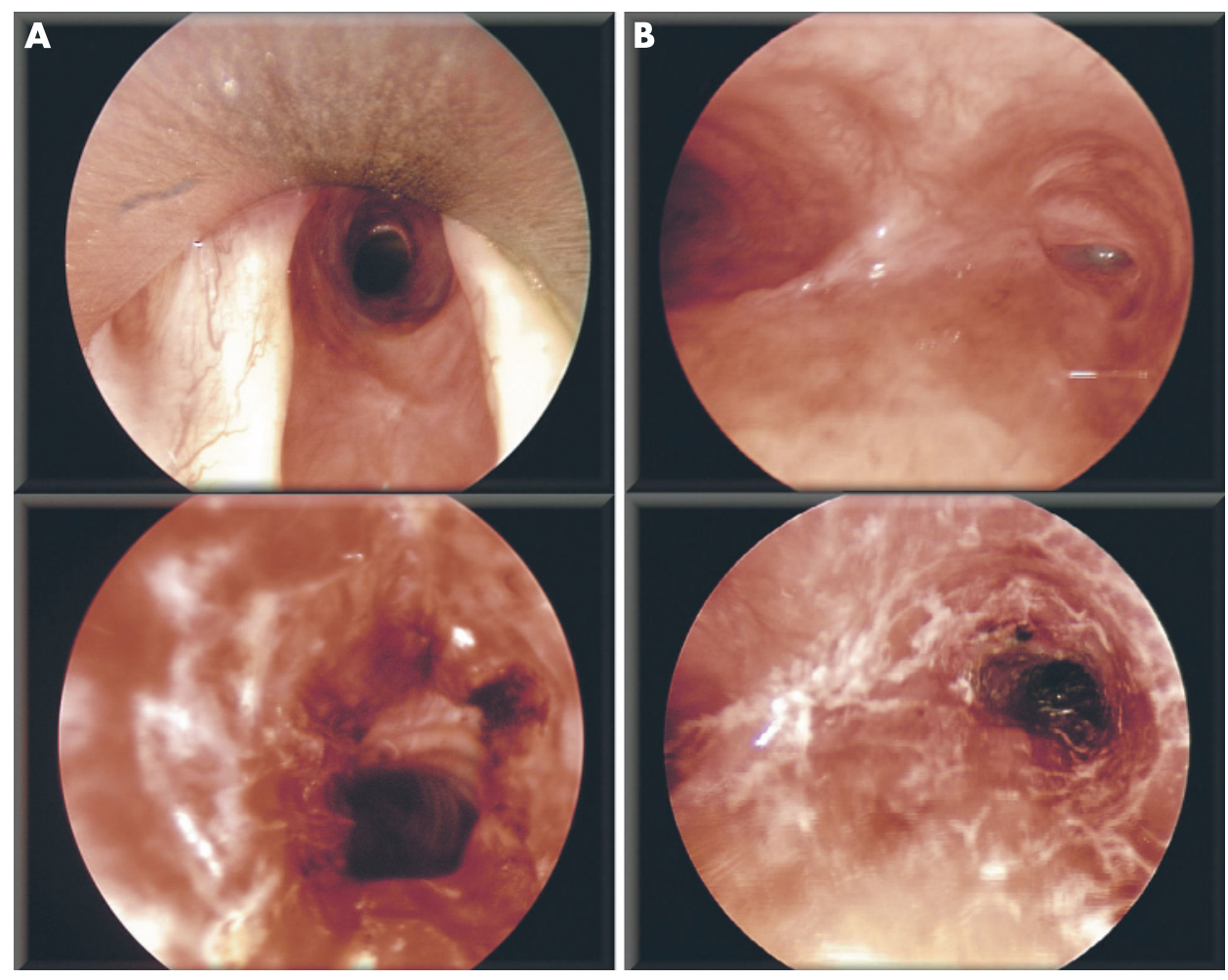

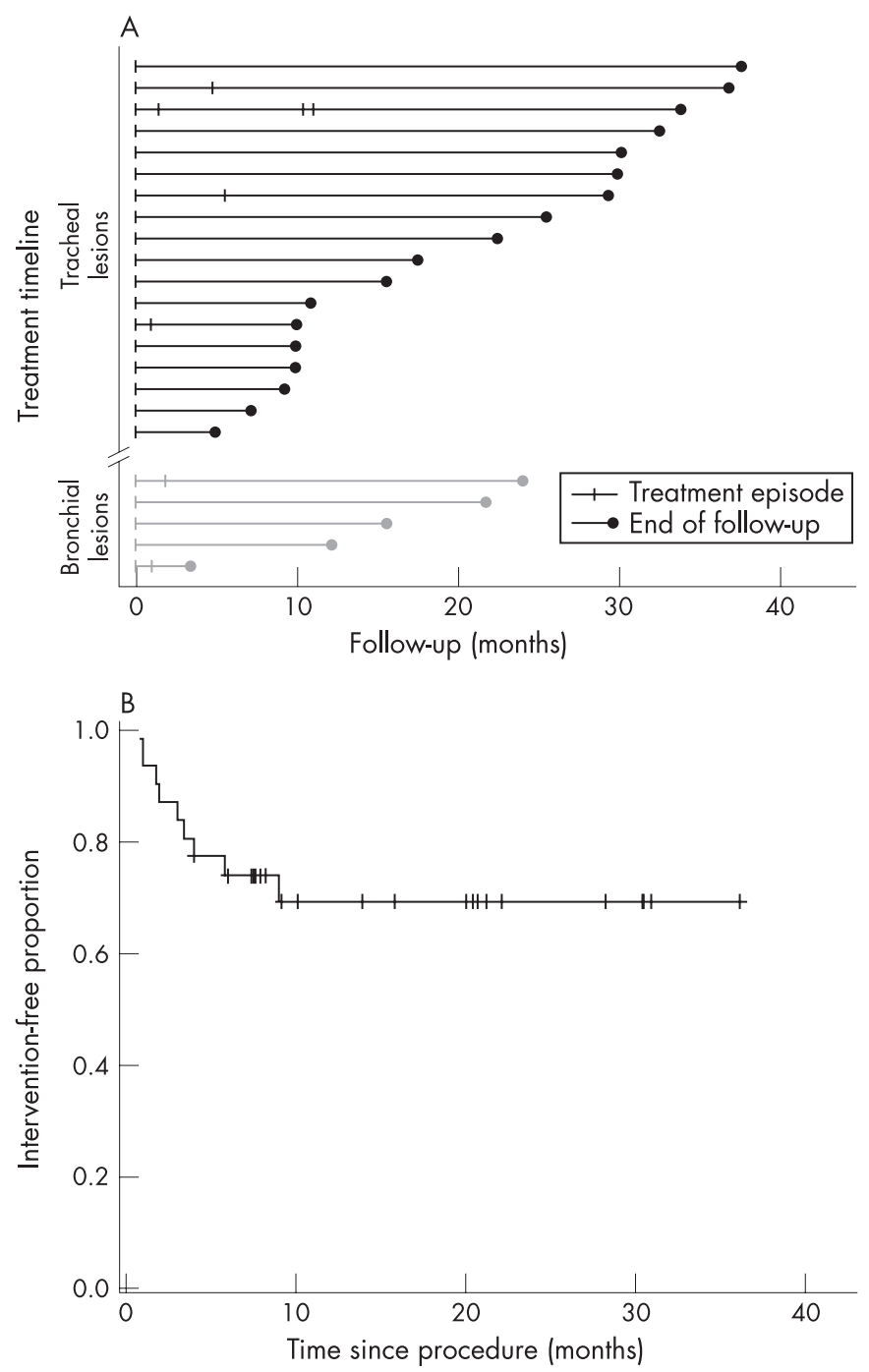

Figure 2 (A) Timeline plot detailing treatments received by individual patients. (B) Kaplan-Meier plot of intervention-free intervals following endoscopic treatment of active tracheobronchial Wegener's granulomatosis.

\section{DISCUSSION}

These findings suggest that airway compromise due to active obstructive tracheobronchial WG can be satisfactorily managed with endoscopic surgery, intralesional steroid therapy and balloon dilatation. These results are in accordance with the experience of Hoffman and colleagues ${ }^{17}$ and Langford and colleagues $^{11}$ who studied subglottic stenosis due to WG. The present work extends the application of this treatment approach to bronchial lesions and differs from the above studies in excluding revision cases with mature fibrotic scars, focusing instead on patients with airway compromise due to active tracheobronchial WG without prior treatment.

There exists a divergence of opinion regarding the optimal treatment of active obstructive tracheobronchial WG. The traditional view, which continues to be advocated, ${ }^{18}$ has held that, with the exception of life threatening lesions, which need to be bypassed with a tracheostomy or opened with a stent, ${ }^{19}$ active obstructive WG should not be approached surgically. Surgery should instead be directed towards decannulation and reconstruction of mature airway scars, once active inflammation has been brought under control.
This is a treatment strategy with which the present authors cannot agree, given that tracheobronchial WG poses a serious, but essentially localised, problem which has an underlying inflammatory cause. Given the advances in shared airway surgery and anaesthesia, the acute obstructive component of this problem can be treated with minimally invasive surgery and dilatation. Furthermore, evidence is accumulating to suggest that the underlying inflammation can be adequately treated with high dose intralesional steroid therapy. ${ }^{11} 1517$ It was possible, using this approach, to reduce the requirement for placing a tracheostomy from $40-50 \%{ }^{12}$ to zero.

Current medical management of WG is based on classifying patients into severe generalised, early systemic or limited disease, the first defined as disease which poses an acute threat to life or function of a vital organ, and the last as disease manifestations that do not pose such threats. ${ }^{3}$ Treatment of severe generalised disease necessitates induction immunosuppression with steroids and cyclophosphamide, ${ }^{8}$ while early systemic disease may be managed with steroids and methotrexate. ${ }^{7}$ Patients with obstructive tracheobronchial WG may be classified as having severe disease, since airway obstruction impedes ventilation and mucociliary clearance, causing ventilatory insufficiency and pulmonary infections, respectively, which can be acutely life threatening. The findings of the present study together with those of Hoffman et al and Langford and colleagues ${ }^{11} 17$ suggest that local treatment of airway obstruction can effectively downstage the disease from severe to limited. Tracheobronchial WG often runs a course independent of other manifestations of WG, ${ }^{11}$ and effective local treatment of airway lesions could lead to early tapering of systemic immunosuppression in selected patients. This multidisciplinary treatment strategy, which is consistent with earlier recommendations, ${ }^{11}{ }^{17}$ was deployed in 1-2 patients towards the end of the present study, and a further prospective study of the impact of a multidisciplinary approach to treating obstructive tracheobronchial WG on the dose and duration of systemic therapy is currently underway. Moreover, in those patients whose disease activity at distant sites necessitates continuing immunosuppression, treatment of tracheobronchial lesions leads not only to symptomatic benefit but also, by improving mucociliary clearance, reduces the likelihood of pulmonary infections developing as a sequelae of retention of secretions in immunocompromised individuals.

None of the patients in the present series required a tracheostomy or a stent, a finding that is in keeping with previous observations. ${ }^{11}$ We would be very reluctant indeed to perform a tracheostomy or a stent procedure on any patient with tracheobronchial WG, given that even patients with severe airway obstruction can be safely anaesthetised and treated with specialised shared airway techniques. We acknowledge however that both of these procedures will continue to be performed on patients who acutely present with life threatening airway compromise locally, who may not be safe for transfer to the nearest airway unit. In these cases, we recommend referral to an airway unit once the obstruction has been safely bypassed so that lesions can be treated and the patient decannulated. For those patients who do require a stent, we urge that whenever possible, a removable stent such as the Aero tracheobronchial stent (Alveolus Inc, Charlotte, North Carolina, USA) be deployed, and removed at the earliest opportunity. If this is not locally available and a permanent stent is deployed as a life saving procedure, the patient should, in our view, be rapidly referred to an airway unit so that the stent can be removed before it is encased in granulation and scar tissue. There is no 
justification for placing and retaining a permanent stent for a benign disease with a favourable long term prognosis ${ }^{2}$ given the significant long term complications that are associated with the placement of permanent stents for benign conditions. ${ }^{13} 14$

In conclusion, the findings of this study suggest that active obstructive tracheobronchial WG can be satisfactorily managed with minimally invasive surgery undertaken using specialist shared airway techniques, obviating the need for airway bypass surgery or stenting, and preventing the long term sequelae of chronic airway scarring. Given the high incidence of tracheobronchial involvement in WG, we suggest that all patients with unexplained pulmonary symptoms should be screened for central airway stenosis with flow-volume loops as part of their diagnostic investigation, and a physician led coordinated multidisciplinary approach to the management of tracheobronchial WG should be adopted. Surgery for this condition should be conservative and aimed at relieving mechanical obstruction and delivering intralesional steroids. Wegener's lesions are benign and transitory and, in our view, carrying out tracheostomies, placing permanent stents or performing complete circumferential endoluminal resections to achieve "normal" intraoperative airway appearances are strongly contraindicated in this condition.

Competing interests: SARN, DJH and GSS have received a research grant from Alveolus Inc, which is a manufacturer of removable pulmonary stents for work unrelated to this project. Although our paper argues against the use of any stents in Wegener's, we have discussed a proviso that if stenting does need to be done as the only available life saving procedure, then a removable stent must be used (for reasons laid out in the manuscript). As such, we feel that in the spirit of full transparency, the grant we have received from Alveolus, although unrelated to the present study, needs to be declared.

\section{REFERENCES}

1. Jennette JC, Falk RJ. Small-vessel vasculitis. N Engl J Med 1997;337:1512-23.

2. Hoffman GS, Kerr GS, Leavitt RY, et al. Wegener granulomatosis: an analysis of 158 patients. Ann Intern Med 1992;116:488-98.
3. Seo $\mathbf{P}$, Stone JH. The antineutrophil cytoplasmic antibody-associated vasculitides. Am J Med 2004;117:39-50.

4. Wegener's Granulomatosis Etanercept Trial (WGET) Research Group Etanercept plus standard therapy for Wegener's granulomatosis. N Engl J Med 2005;352:351-61.

5. Walton EW. Giant-cell granuloma of the respiratory tract (Wegener's granulomatosis). Br Med J 1958;2:265-70.

6. Fauci AS, Haynes BF, Katz $\mathrm{P}$, et al. Wegener's granulomatosis: prospective clinical and therapeutic experience with 85 patients for 21 years. Ann Intern Med 1983;98:76-85.

7. de Groot K, Rasmussen N, Bacon PA, et al. Randomized trial of cyclophosphamide versus methotrexate for induction of remission in early systemic antineutrophil cytoplasmic antibody-associated vasculitis. Arthritis Rheum 2005;52:2461-9.

8. Jayne D, Rasmussen N, Andrassy K, et al. European Vasculitis Study Group. A randomized trial of maintenance therapy for vasculitis associated with antineutrophil cytoplasmic autoantibodies. N Engl J Med 2003;349:36-44

9. Little MA, Pusey C. Glomerulonephritis due to antineutrophil cytoplasm antibodyassociated vasculitis: an update on approaches to management. Nephrology 2005; 10:368-76.

10. de Groot $\mathbf{K}$, Jayne D. What is new in the therapy of ANCA-associated vasculitides? Take home messages from the 12th workshop on ANCA and systemic vasculitides. Clin Nephrol 2005;64:480-4.

11. Langford CA, Sneller MC, Hallahan CW, et al. Clinical features and therapeutic management of subglottic stenosis in patients with Wegener's granulomatosis. Arthritis Rheum 1996;39:1754-60.

12. Lebovics RS, Hoffman GS, Leavitt RY, et al. The management of subglottic stenosis in patients with Wegener's granulomatosis. Laryngoscope 1992; 102:1341-5.

13. Eller RL, Livingston WJ, Morgan CE, et al. Expandable tracheal stenting for benign disease: worth the complications? Ann Otol Rhinol Laryngol 2006;115:247-52.

14. Gaissert HA, Grillo HC, Wright CD, et al. Complication of benign tracheobronchial strictures by self-expanding metal stents. J Thorac Cardiovasc Surg 2003;126:744-7.

15. Nouraei SAR, Singh A, Patel A, et al. Early endoscopic treatment of acute inflammatory airway lesions improves the outcome of postintubation airway stenosis. Laryngoscope 2006;116:1417-21.

16. Myer CM, O'Connor DM, Cotton RT. Proposed grading system for subglottic stenosis based on endotracheal tube sizes. Ann Otol Rhinol Laryngol 1994;103:319-23.

17. Hoffman GS, Thomas-Golbanov CK, Chan J, et al. Treatment of subglottic stenosis, due to Wegener's granulomatosis, with intralesional corticosteroids and dilation. $J$ Rheumatol 2003;30:1017-21.

18. Gluth MB, Shinners PA, Kasperbauer JL. Subglottic stenosis associated with Wegener's granulomatosis. Laryngoscope 2003;113:1304-7.

19. Watters K, Russel J. Subglottic stenosis in Wegener's granulomatosis and the Nitinol stent. Laryngoscope 2003;113:2222-4.

Access the latest content chosen by our Editors

BMJ Journals editors select an article from each issue to be made free online immediately on publication. Other material is free after 12 months to non-subscribers. Access the Editor's Choice from the home page - or expand your horizons and see what the other BMJ Journals editors have chosen by following the links on any BMJ Journal home page. 